\title{
Epithelial shedding is associated with nasal reactions to cold, dry air
}

\author{
Alvaro A. Cruz, MD, ${ }^{\mathrm{a}, \mathrm{b}}$ Robert M. Naclerio, MD, ${ }^{\mathrm{a}, \mathrm{c}}$ David Proud, PhD, ${ }^{\mathrm{a}, \mathrm{d}}$ and \\ Alkis Togias, $\mathbf{M D}^{\mathbf{a}, \mathbf{e}}$ Baltimore, Md, Bahia, Brazil, Chicago, Ill, and Calgary, Alberta, Canada
}

\begin{abstract}
Background: Cold, dry air (CDA) can cause symptoms of rhinitis and obstructive airway responses. The pathophysiology of these reactions is not understood. One hypothesis is that the respiratory mucosa of individuals with CDA sensitivity cannot compensate for the loss of water that occurs on exposure to the stimulus, leading to epithelial damage.
\end{abstract}

Objective: To test for an association between nasal reactions to CDA and the number of epithelial cells recovered in nasal fluids. Methods: Ten CDA-sensitive subjects received nasal provocations with CDA and warm, moist air; 10 CDAinsensitive subjects received CDA; and 10 subjects with allergic rhinitis received allergen and diluent challenges. Nasal lavage cytology was performed at baseline and after the challenge. Symptoms were recorded and histamine, $\left[{ }^{3} \mathrm{H}\right]-\mathrm{N}-\alpha$-tosyl-Larginine methyl ester-esterase activity, tryptase, and albumin were assayed in nasal lavages.

Results: A 6-fold increase in nasal lavage epithelial cells was found in the CDA-sensitive group after CDA $(P<.01)$, but not after warm, moist air. No changes were observed in the CDAinsensitive group, or after allergen or diluent in allergic rhinitis. Conclusion: Epithelial cell shedding accompanies clinical responses to CDA in the human nose. This supports the hypothesis that the airway mucosa of CDA-sensitive individuals

From a the Johns Hopkins Asthma and Allergy Center, Johns Hopkins University, Baltimore; 'be Department of Medicine, Pulmonary and Immunology Divisions, Federal University of Bahia School of Medicine; ${ }^{c}$ the Section of Otolaryngology, Head and Neck Surgery, Department of Surgery, University of Chicago; the Department of Physiology and Biophysics, University of Calgary; and ${ }^{e}$ the Division of Allergy and Clinical Immunology and Division of Pulmonary and Critical Care Medicine, Johns Hopkins University School of Medicine, Baltimore.

Supported by grants PO1 AI 37163, RO1DC02714, and RO1AI08270 from the National Institutes of Health, Bethesda, Md, and from CAPES (Ministry of Education), Brazil.

Disclosure of potential conflict of interest: A. Cruz has received grants from Coordenação de Aperfeiçoamento de Pessoal de Nível Superior and Conselho Nacional de Desenvolvimento Científico e Tecnológico, Federal Government of Brazil. R. Naclerio has consultant arrangements with Aventis, Merck, Schering, GlaxoSmithKline, Altana, and Dey; has received grants from GlaxoSmithKline, Merck, Corixa, and Novartis; and is on the speakers' bureau for Merck and Aventis. D. Proud has received grants from the Canadian Institutes of Health Research and Procter \& Gamble.

The rest of the authors have declared that they have no conflict of interest.

Received for publication July 18, 2005; revised January 26, 2006; accepted for publication January 31, 2006.

Available online May 2, 2006

Reprint requests: Alkis Togias, MD, Johns Hopkins Asthma and Allergy Center, 5501 Hopkins Bayview Circle, Unit Office 7, Baltimore, MD 21224-6801. E-mail: atogias@jhmi.edu.

$0091-6749 / \$ 32.00$

(c) 2006 American Academy of Allergy, Asthma and Immunology doi:10.1016/j.jaci.2006.01.054 cannot compensate for the water loss that occurs under extreme conditions leading to epithelial damage. Clinical implications: A defect in mucosal water homeostasis may need to be considered in individuals who get excessive nasal symptoms when exposed to cold and dry, windy environment. (J Allergy Clin Immunol 2006;117:1351-8.)

Key words: Hyperosmolarity, hypertonicity, nasal allergen provocation, nasal lavage, nasal challenge

Nasal sensitivity to cold, dry air (CDA) manifests with symptoms of rhinitis including profuse rhinorrhea and nasal congestion. ${ }^{1}$ These symptoms can be reproduced in the laboratory with CDA provocation. ${ }^{2}$ Some individuals are exquisitely sensitive to this stimulus and, as a group, patients with nonallergic rhinitis react to CDA more vigorously than healthy controls. ${ }^{3}$ CDA sensitivity is also prominent in skiers, probably because, when descending slopes at high speed, they get exposed to a stimulus of high magnitude. ${ }^{4}$ Particular interest in the nasal reaction to CDA exists because understanding of its pathophysiology may offer insights into the mechanisms of lower airway reactions to the same stimulus that are quite prominent in asthma.

Early on in the development of the nasal CDA provocation model, it became clear that the best predictor of a nasal reaction to CDA in the laboratory is a clinical history of sensitivity to the natural stimulus. Individuals with severe nasal symptoms in cold, windy weather develop symptoms with experimental provocation and their nasal secretions after exposure to CDA contain increased levels of histamine, sulfidopeptide leukotrienes, and tryptase, ${ }^{5,6}$ suggestive of mast cell activation. Also, sensory nerves are involved in the reaction, because provocation through 1 nostril results in bilateral responses. ${ }^{7}$ In contrast, individuals who deny nasal symptoms when exposed to cold, windy weather have no clinical response to CDA provocation and show no evidence of mast cell mediator release or neuronal activation.

The mechanistic basis of the difference between CDAsensitive and insensitive individuals is unknown. We have previously demonstrated that neither the presence of atopy nor nasal responsiveness to histamine predicts CDA responsiveness. ${ }^{8}$ In a limited number of experiments, we have found that the osmolarity of the epithelial lining fluid is increased after CDA provocation in the CDA-sensitive 
Abbreviations used

CDA: Cold, dry air

WMA: Warm, moist air

but not in the insensitive group. ${ }^{9}$ Also, when both groups undergo nasal challenge with a hyperosmolar solution, CDA-sensitive subjects release significantly more histamine in nasal lavage fluids compared with CDA-insensitive subjects. ${ }^{8}$ These observations led us to the general hypothesis that the underlying difference between CDA-sensitive and insensitive individuals relates to the ability of the mucosa to cope with conditions that demand increased water supply to inhaled air or to the epithelial surface, whether inhalation of dry air or application of a hyperosmolar stimulus. If a defect in compensating for water loss exists in the CDA-sensitive individuals, breathing CDA may lead to hypertonicity of the epithelial layer and possibly of the superficial submucosal tissue, resulting in sensory nerve stimulation ${ }^{10,11}$ and mast cell activation. ${ }^{12,13}$ At the same time, the epithelium can be subject to damage from desiccation and detachment. If, on the other hand, water supply to the epithelial surface under stressful conditions is ample, none of these phenomena should take place, and the subject undergoing CDA provocation should have no reaction to the stimulus.

We conducted this study to test part of this hypothesis, whether epithelial shedding takes place after CDA nasal challenge and whether this is more prominent in the CDAsensitive group. Because of the possibility that epithelial shedding may not necessarily be the result of desiccation but of an acute toxic effect of inflammatory mediators on epithelial cells, we added a control group in this study, a group of individuals with allergic rhinitis who received nasal challenges with allergen or its vehicle. Our rationale for this control was that, because the pattern of inflammatory mediator release that is observed in nasal lavage fluids after allergen and CDA challenges is similar, ${ }^{2,6,14}$ if these products were the cause of epithelial shedding, epithelial cells in nasal lavage fluids would be found in similar numbers after CDA and allergen challenges.

\section{METHODS \\ Subjects}

We studied 3 groups, 10 volunteers each, ages 20 to 46 years. CDA-sensitive subjects reported rhinorrhea with cold and windy weather and had a positive reaction to a previous CDA challenge. ${ }^{2}$ Six of them also had allergic rhinitis. CDA-insensitive subjects reported no symptoms in cold weather and had a previous negative CDA provocation. Four had allergic rhinitis. Volunteers with allergic rhinitis had positive skin tests to grass or ragweed and were tested when asymptomatic, outside pollen seasons. Two of the subjects in the CDA-sensitive group and 3 in the CDA-insensitive group also participated in the allergic rhinitis group. All subjects gave informed consent, and the study was approved by the Johns Hopkins Joint Committee on Clinical Investigation.

\section{Nasal challenges}

Fig 1 describes the various provocation protocols in which each group of study subjects participated. CDA-sensitive and CDA-insensitive subjects received a challenge with CDA. The CDA-sensitive group was also challenged with warm, moist air (WMA) to control for the mechanical effects of breathing air through the nose. The allergic rhinitis group received 1 provocation with allergen and 1 with diluent. CDA and WMA provocations were performed as previously described. ${ }^{2,5,9}$ Allergen challenges were performed with short ragweed or a mixture of grasses, also with a previously described methodology. ${ }^{15}$ Fifty protein nitrogen units of the respective allergen extract were sprayed into each nostril. Within each group, challenges were performed in random order. The minimum period between challenges after WMA or diluent was 48 hours, and after CDA or allergen, 5 days.

\section{Nasal lavages and lavage outcomes evaluations}

Nasal lavages were also performed as previously described. ${ }^{2,14}$ In each protocol, evaluations of the returned lavage fluids were performed on 4 occasions, PRE A, PRE B, BASELINE, and POST challenge lavages (Fig 1). A set of preparatory (PRE) lavages was first performed to clear pre-existing cells and mediators. Baseline lavages were performed 1 hour before nasal challenge, so that ample time was given for the hydration state of the mucosa to return to its prelavage condition. The time interval between baseline and postchallenge lavages was equal to the interval between the preparatory and baseline lavages (Fig 1). This was meant to control for outcome changes after the respective provocation, reflecting a spontaneous process. On each lavage occasion, we used a pair of lavages, the first with 5 and the second with $10 \mathrm{~mL}$ lactated Ringer's warmed to body temperature and divided into the 2 nostrils. Fluids from the 5 -mL lavages were assayed for mediators and biologic markers. Measurements of histamine, ${ }^{16}$ $\left[{ }^{3} \mathrm{H}\right]-\mathrm{N}-\alpha$-tosyl-L-arginine methyl ester (TAME)-esterase activity, ${ }^{17,18}$ tryptase,${ }^{6}$ and albumin ${ }^{19}$ were made by using established assays. The cell pellets from each lavage pair were combined, total cell numbers were obtained with the use of a hemocytometer, and cytospin slides (Shandon, Sewickley, Pa) for differentials were generated. Differential counts were performed after staining with Diff-Quick (American Scientific, McGaw Park, Ill). ${ }^{20,21}$ Squamous, basal, and columnar epithelial cells were counted on the stained slides as a single cellular category. Cell differentials were conducted on coded slides.

\section{Symptom scores}

Rhinorrhea and nasal obstruction were self-evaluated on 10-cmlong visual analogue scales marked at each end with "no symptoms" and "the worst it has ever been." 22 Evaluations took place at the beginning of each protocol (PRE A) and at the time points when the PRE B, BASELINE, and POST lavage sessions were performed (Fig 1).

\section{Data analysis}

The primary outcome of this study was the absolute number of epithelial cells in nasal lavage fluids. However, because the volume of returned lavage fluids in the CDA-sensitive subjects was higher after the CDA challenge compared with the other time points, we decided also to examine the number of epithelial cells per returned lavage volume unit $(\mathrm{mL})$. Because the data distribution was not normal, nonparametric statistics were used, and the results are presented as median values with interquartile ranges. Friedman ANOVA was conducted within each protocol to examine the effect of CDA or allergen in comparison with prechallenge values or to values obtained after the respective negative controls. Post hoc analysis 
cold, dry air sensitive subjects $(n=10)$

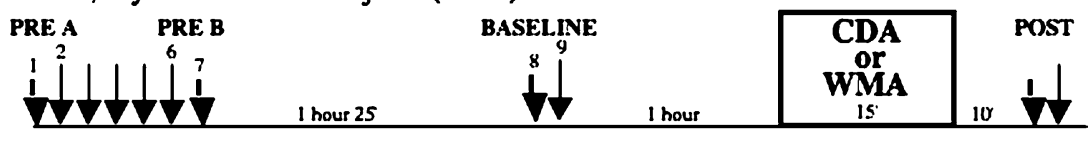

cold, dry air insensitive subjects $(n=10)$

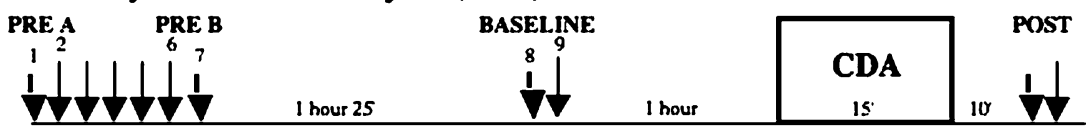

allergic rhinitis subjects $(n=10)$

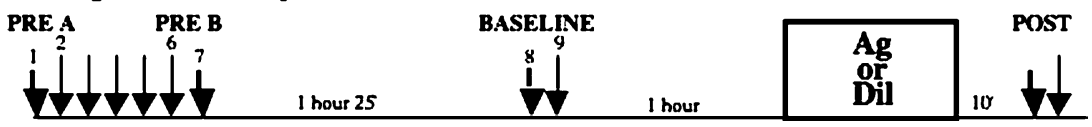

FIG 1. Illustration of the various nasal provocation protocols used in this study. The top pane/presents the protocols for the WMA or CDA provocations performed on the $10 \mathrm{CDA}$-sensitive subjects. The middle panel presents the protocol for the CDA challenge performed on the 10 CDA-insensitive subjects. The bottom panel presents the protocols for the allergen $(\mathrm{Ag})$ and diluent (Dil) challenges performed on the 10 subjects with allergic rhinitis. Whereas the duration of CDA or WMA challenges was 15 minutes, only a few seconds were required for $\mathrm{Ag}$ or Dil to be administered into the nasal cavities. Arrows represent nasal lavages (short arrows indicate lavages with $5 \mathrm{~mL}$ Lactated Ringer, whereas tall arrows indicate lavages with $10 \mathrm{~mL}$ ). For cell counts and differentials, pairs of returned lavage fluids were analyzed. PRE A, lavages 1 and 2; PRE $B$, lavages 6 and 7; BASELINE, lavages 8 and 9; POST, the 2 lavages performed 10 minutes after the respective nasal provocation.

was conducted with the use of the Wilcoxon matched-pairs, signedranks test. Kruskal-Wallis ANOVA followed by the Mann-Whitney $U$ test was used to compare the changes from prechallenge values induced by CDA in the CDA-sensitive and the CDA-insensitive groups and by allergen in the group of subjects with allergic rhinitis. Twotailed $P$ values $<.05$ were considered statistically significant.

\section{RESULTS}

\section{Epithelial cells}

Fig 2 depicts the primary outcome, epithelial cell data expressed as absolute numbers of cells in returned nasal lavage fluids. In Fig 2, $A$, the results from the challenges with CDA and WMA performed on the CDA-sensitive subjects are presented. Many epithelial cells were found in the returned fluids from the lavages that initiated each protocol (PRE A). The preparatory lavages reduced the number of epithelial cells (PRE B), and the 85-minute waiting period did not result in any spontaneous increase. Thus, BASELINE values can be considered stable within the time limits of these experiments. CDA provocation resulted in a statistically significant 6 -fold increase over baseline in total epithelial cells in the POST challenge lavages (Friedman ANOVA for all 4 time points, $P=.006$; Wilcoxon test for BASELINE vs POST, $P=.008$ ). The findings were consistent when the analysis was performed on the "epithelial cells/mL" outcome (Wilcoxon test for BASELINE vs POST, $P=.007$ ), which we thought appropriate to examine as an additional outcome because the volume of the returned lavage differed among the 4 time points of this protocol (Friedman ANOVA, $P=$ $.01)$. In contrast with the CDA results, WMA challenge did not induce an increase in epithelial cells from baseline and a trend toward reduction was observed (Friedman ANOVA, $P=.08$ ). Fig $2, B$, presents the results of the CDA provocation performed on the CDA-insensitive subjects. In contrast with the CDA-sensitive group, CDA had no significant effect on epithelial cell numbers in nasal lavage fluids in these individuals (Friedman ANOVA was significant in this protocol, $P=.03$, but the difference between the BASELINE and the POST sample was not: Wilcoxon test, $P=.17$ ). Finally, Fig 2, $C$, depicts the results obtained from the group of subjects with allergic rhinitis during their allergen and diluent nasal provocations. In both protocols, epithelial cell numbers did not differ across the 4 time points (Friedman ANOVA, $P=.17$ and $P=.27$ for allergen and diluent, respectively).

Statistically significant differences among the 5 protocols were obtained when the analysis was performed on the changes in total epithelial cell numbers from BASELINE to the POST challenge lavages (KruskalWallis ANOVA, $P=.02$; data not shown). In this analysis, the CDA effect in the CDA-sensitive group was stronger than that of WMA (Wilcoxon test, $P=.02$ ) and than that of the allergen provocation in the allergic rhinitis group (Mann-Whitney $U$ test, $P=.047$ ). The difference between the CDA-sensitive and insensitive individuals in terms of the CDA-induced change in epithelial cells from baseline did not reach statistical significance (Mann-Whitney $U$ test, $P=.06$ ). However, as shown in Fig 3, when the analysis was performed on the "epithelial cells/mL" outcome, the CDA challenge in the CDAsensitive subjects resulted in significantly higher values 


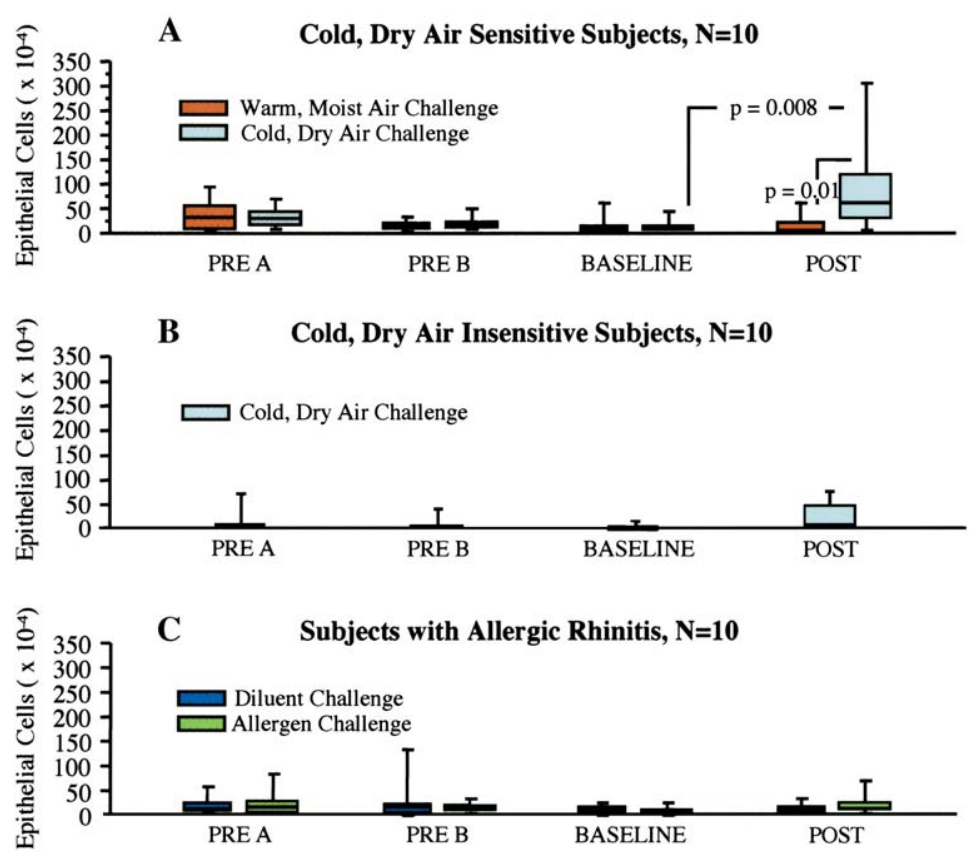

FIG 2. Absolute numbers of epithelial cells in returned nasal lavage fluids. Data are presented as box plots with the middle horizontal lines indicating medians, the top and bottom of each box indicating the 75th and 25th percentiles, respectively, and the top and bottom of the error bars indicating the 90th and 10th percentiles, respectively. A, Data from the WMA and the CDA challenges performed on the group of CDA-sensitive subjects. B, Data from the CDA challenge performed on the group of CDA-insensitive subjects. C, Data from the allergen and diluent challenges performed on the group of subjects with allergic rhinitis. PRE $A$, Data obtained from the combined 2 initial lavages; PRE B, data obtained from the combined 6 th and 7 th initial lavages; BASELINE, data obtained from the combined 8th and 9th lavages, 1 hour before the initiation of the challenge; POST, data obtained from the 2 lavages performed 10 minutes after the end of the respective provocation (see Fig 1 for a more illustrative description of the protocols and the lavage sessions). The vertical axis label indicates that the number shown has to be multiplied by 10,000 for derivation of the total cell number.

compared with each of the other 4 protocols, including the CDA challenge in the CDA-insensitive group $(P=.03)$. Also with this analysis, within the group of subjects with allergic rhinitis, the allergen and diluent-induced epithelial cell/mL changes from BASELINE were not different (Wilcoxon test, $P=.26$; Fig 3).

\section{Leukocytes}

As expected, in contrast with epithelial cells, statistically significant differences between groups in total leukocytes were not detected either in the initiating lavages or after the various provocations. Overall, there was a trend for total leukocytes to be lower in all lavage fluids of the CDA-insensitive individuals compared with the CDAsensitive subjects and the subjects with allergic rhinitis. This trend almost reached statistical significance in the POST challenge lavages (Kruskal-Wallis ANOVA, $P=$ $.05)$. When the leukocyte differential counts were considered, CDA-insensitive individuals proved to have significantly lower eosinophils at every time point compared with the other 2 groups (data not shown). However, no difference was observed between CDA-sensitive and allergic rhinitis subjects. Within each protocol, 10 minutes after the respective challenges, no significant changes over baseline in the total number of leukocytes or in polymorphonuclear cells, eosinophils, and nonepithelial mononuclear cells were found. Comparison of the 5 protocols in terms of the challenge-induced change from baseline in total leukocyte or in eosinophil counts failed to demonstrate any differences (Kruskal-Wallis ANOVA, $P=.36$ and $P=.41$, respectively; data not shown).

\section{Symptoms and biologic markers}

Subjects sensitive to CDA challenged with CDA, as well as subjects with allergic rhinitis challenged with allergen, developed rhinorrhea and nasal congestion (data not shown) accompanied by significant increases over BASELINE in the levels of albumin, TAME-esterase activity, histamine, and tryptase in the POST challenge nasal lavage fluids (Fig 4).

When the nasal provocation-induced changes from baseline were considered, the CDA challenge of CDAsensitive individuals and the allergen challenge of the subjects with allergic rhinitis were not statistically different in any biologic marker or symptom. On the other hand, the changes induced by CDA provocation in the CDA-sensitive group were significantly higher than those induced by WMA in the same group (with the exception of histamine), and by CDA in the CDAinsensitive subjects (data not shown). Similarly, the 


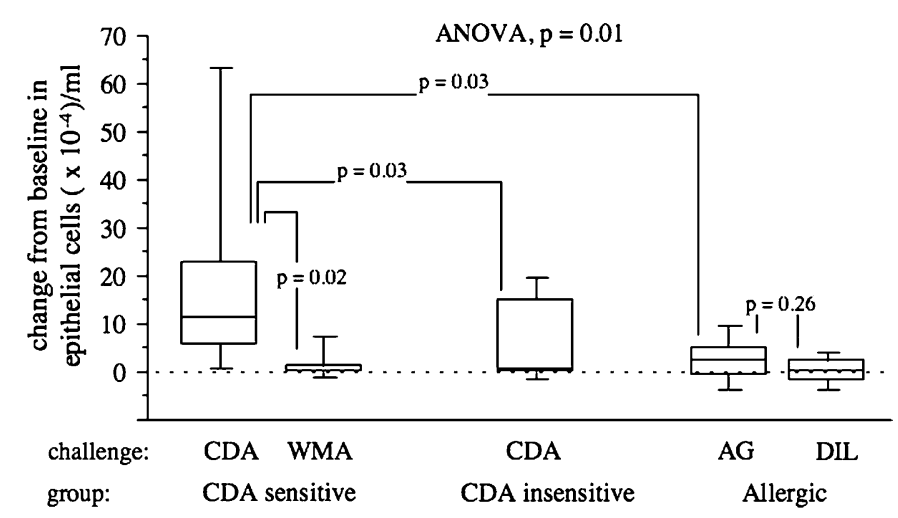

FIG 3. Changes from baseline after various nasal provocations in epithelial cell numbers per milliliter of returned nasal lavage fluids. Data are presented as box plots with the middle horizontal lines indicating medians, the top and bottom of each box indicating the 75th and 25th percentiles, respectively, and the top and bottom of the error bars indicating the 90th and 10th percentiles, respectively. AG, Allergen; DIL, diluent. The vertical axis label indicates that the number shown has to be multiplied by 10,000 for derivation of the total cell number.

Cold, Dry Air Challenge (cold, dry air-sensitive subjects, $\mathrm{N}=10$ )

Allergen Challenge (subjects with allergic rhinitis, $\mathrm{N}=10$ )
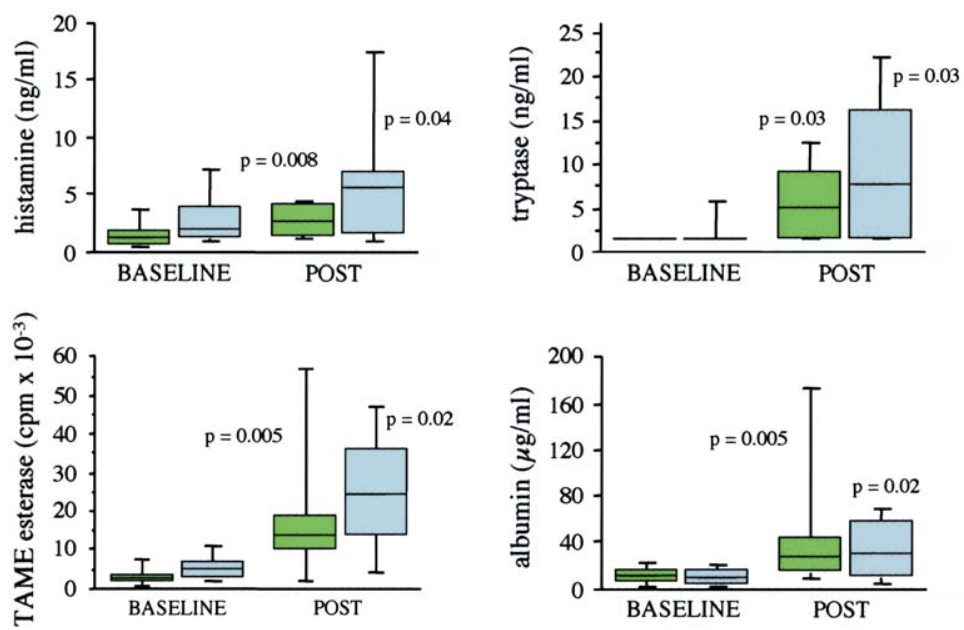

FIG 4. Concentrations of inflammatory mediators and reaction markers in nasal lavage fluids at baseline and 10 minutes after the CDA provocation in CDA-sensitive subjects and after the allergen provocation in subjects with allergic rhinitis (POST). Data are presented as box plots with the middle horizontal lines indicating medians, the top and bottom of each box indicating the 75th and 25th percentiles, respectively, and the top and bottom of the error bars indicating the 90 th and 10th percentiles, respectively. $P$ values indicate the level of statistical significance for the comparison of the POST to the respective BASELINE values.

changes in all symptoms and mediators/markers induced by allergen in the allergic rhinitis group were significantly higher compared with those induced by the diluent challenge.

\section{DISCUSSION}

This study was designed to test the hypothesis that, with CDA provocation in the human nose, epithelial cell shedding occurs in individuals who develop rhinitis symptoms. This hypothesis is unequivocally supported by our findings. In this group of individuals, total epithelial cells as well as the number of epithelial cells per milliliter of returned nasal lavage fluid were significantly increased over baseline 10 minutes after the completion of nasal CDA provocation. Importantly, several negative controls that were incorporated into this study's protocol provided strong evidence that the cellular shedding detected after the CDA provocation is specific for CDA and primarily detectable in the group of subjects who have nasal symptoms with this stimulus. These controls 
included a nasal provocation with WMA in the CDAsensitive individuals, a nasal provocation with CDA in a group of subjects who had no clinical CDA sensitivity, and a nasal allergen and diluent provocation in a group of subjects with seasonal allergic rhinitis, examined while out of pollen season.

The allergen provocation was designed to match the CDA provocation with respect to the amount of biochemical markers and mediators released in nasal fluids during the acute mucosal response. Despite this match (Fig 4), allergen challenge was not associated with an increase in epithelial cell numbers in nasal fluids. These results indicate that the inflammatory events observed immediately after CDA provocation (mast cell activation, glandular activation, plasma extravasation) are not responsible for the loss of epithelial cells in CDA-sensitive subjects.

The fact that WMA did not induce any changes in epithelial cells indicates that nasal epithelial shedding in CDA-sensitive individuals does not result from any type of manipulation (nasal lavage, or the sheer force effect of high airflow) and that it is not a result of a spontaneous process. A spontaneous process could have been implied by the trend for increased epithelial cells in CDA-sensitive subjects in the preliminary lavage fluids (Fig 2).

Cold, dry air provocation did not induce statistically significant epithelial shedding when performed on the CDA-insensitive group. This observation supports our view that the clinical response to CDA is linked to some form of a mucosal water transportation defect. This view is based on our previous finding that CDA challenge increases the osmolarity of nasal secretions only in CDAsensitive subjects. ${ }^{9}$ The nature of the alleged defect is unknown. For example, we have no information on electrolyte transportation across the nasal mucosa, nasal potential differences, tight junction transport, or aquaporin function in CDA-sensitive individuals. However, if a defect in 1 or more of these functions were present, it could offer the basis for understanding the current findings. High demand for water from the mucosa would lead to hypertonicity of nasal fluids and, consequently, to alterations of the cytoskeleton, with disruption of the links between intermediate filaments and the proteins that contribute to the integrity of desmosomal and/or hemidesmosomal connections. This could lead to epithelial cell detachment. It is quite possible that the mechanical effect of breathing air at high flow $(25 \mathrm{~L} / \mathrm{min})$ contributes to the shedding of epithelium, yet the results from the WMA challenge clearly show that high airflow cannot produce shedding on its own.

An interesting observation was that the number of eosinophils in the preliminary lavages of CDA-sensitive subjects was higher than that of the CDA-insensitive ones. Given the widely acknowledged hypothesis that eosinophil activation may cause airway epithelial damage, ${ }^{23}$ it could be argued that eosinophils in CDA-sensitive subjects were somehow related to the epithelial shedding. However, we found no correlation between preliminary lavage eosinophils and CDA-induced increase in nasal lavage epithelial cells in this group (data not shown).
Is epithelial shedding the cause of the nasal symptoms that occur with CDA provocation? These symptoms may be induced by the release of mast cell products ${ }^{2,5,6}$ or the activation of sensory nerve endings with resultant nasonasal reflexes ${ }^{7}$ and/or release of sensorineural inflammatory peptides. ${ }^{24}$ Our previous attempts to inhibit the symptomatic response to CDA with topical or oral antihistamines have failed ${ }^{25}$ suggesting that histamine release may not have a true pathogenetic role in this reaction. On the other hand, local anesthesia has reduced the secretory response to CDA provocation, ${ }^{7}$ supporting a sensory nerve activation and reflex rhinorrhea scenario. Epithelial shedding can be viewed as a form of trauma to the nasal mucosa leading to nociceptor exposure. If so, one can propose a logical link among epithelial shedding, sensorineural activation, and nasal symptoms. However, another mechanism for activation of these sensory nerve endings may be hypertonicity itself, which is known to activate capsaicin-sensitive nerve endings in the human nose directly. ${ }^{10}$ Recently, it has been demonstrated that the primary neural receptor responding to a hypertonic stimulus is transient receptor potential vanilloid 1 , the capsaicin receptor. ${ }^{11}$ Finally, epithelial cells, under a hypertonic stimulus, may release arachidonic acid metabolic products, particularly 15-hydroxyeicosatetraenoic acid, ${ }^{26,27}$ that may activate sensory nerve endings $\mathrm{s}^{28}$ and produce symptoms.

If the theory of mucosal desiccation is correct, we should expect that prehumidification of the nasal mucosa would reduce the reaction to CDA. Indeed, data we previously presented in abstract form show that the longer the period between nasal lavages and a CDA challenge, the stronger the CDA response. ${ }^{29}$ The desiccation theory also raises the question ${ }^{30}$ whether medications that inhibit secretory functions, such as anticholinergics, worsen the CDA response. We have found that topical application of atropine inhibits CDA-induced rhinorrhea, but we do not know whether epithelial shedding is affected. If humidification of inhaled air takes place as a result of water transportation into the airway lumen through the epithelium and if the water in the mucous produced by the glands is not a major contributor to this process, anticholinergics may stop the production of mucus but will have no detrimental effect on the actual hydration status of the superficial epithelial layer. This hypothesis is supported by the work of Assanasen et $\mathrm{al}^{31}$ who have demonstrated that, if anything, nasal ipratropium increases the water content of air in the nasal passages.

What makes the CDA nasal reaction and its pathogenetic hypothesis also interesting is its potential analogy with CDA hyperventilation and hyperosmolar solutioninduced bronchospasm in patients with asthma. ${ }^{32}$ In animal models of dry air-induced bronchospasm, epithelial detachment has been observed after CDA provocation in $\operatorname{dog}^{33-35}$ and in guinea pigs. ${ }^{36}$ Although the relationship between CDA-induced rhinitis and asthma has not been investigated, it is noteworthy that, in our hands, nasal CDA provocation produces stronger biochemical and nasal symptom responses in subjects with allergic rhinitis and asthma compared with individuals with rhinitis 
alone. ${ }^{37}$ Also, we would like to call attention to a study by Assanasen et $\mathrm{al}^{38}$ suggesting that patients with asthma have decreased ability to humidify inhaled air through the nose ${ }^{38}$ and to previously published findings of increased bronchial epithelial cell numbers after CDA hyperventilation-induced bronchospasm in patients with asthma. ${ }^{39}$ Finally, a possibly related phenomenon is the induction of an asthmalike syndrome in high-performance skiers, which has been confirmed by various groups in Scandinavia and has been associated with airways inflammation and the duration of exposure to subfreezing, windy conditions. ${ }^{40-42}$ It would be interesting to know whether the individuals who developed this syndrome had a history of nasal sensitivity to CDA. In dogs, repetitive exposure of peripheral airways to CDA generates eosinophil, mast cell, and neutrophil influx and leads to thickening of the lamina propria, but it is not known whether these changes are associated with the magnitude of epithelial shedding induced by CDA. ${ }^{43}$

In summary, we have shown that epithelial cell detachment occurs in the nasal mucosa of individuals with sensitivity to CDA when they are exposed to this stimulus. Our results indicate that epithelial shedding may not be the consequence of inflammatory mediator release but rather an effect related to mucosal desiccation, perhaps in synergy with high airflow. We suggest that careful evaluation of the homeostatic properties of the airway mucosa, as they pertain to water transportation and tissue hydration, may offer significant insights into the pathogenesis of upper and lower airways reactions to cold air.

\section{REFERENCES}

1. Togias A. Non-allergic rhinitis. In: Mygind N, Naclerio R, Durham S, editors. Rhinitis. New York: Marcel Dekker, Inc; 1998. p. 383-99.

2. Togias A, Naclerio R, Proud D, Fish J, Adkinson N, Kagey-Sobotka A, et al. Nasal challenge with cold, dry air results in the production of inflammatory mediators: possible mast cell involvement. J Clin Invest 1985;76:1375-81.

3. Braat J, Mulder P, Fokkens W, van Wijk R, Rijntjes E. Intranasal cold dry air is superior to histamine challenge in determining the presence and degree of nasal hyperreactivity in nonallergic noninfectious perennial rhinitis. Am J Respir Crit Care Med 1998;157:1748-55.

4. Silvers WS. The skier's nose: a model of cold-induced rhinorrhea. Ann Allergy 1991;67:32-6.

5. Togias AG, Naclerio RM, Peters SP, Nimmagadda I, Proud D, KageySobotka A, et al. Local generation of sulfidopeptide leukotrienes upon nasal provocation with cold, dry air. Am Rev Respir Dis 1986;133: 1133-7.

6. Proud D, Bailey G, Naclerio R, Reynolds C, Cruz A, Eggleston P, et al Tryptase and histamine as markers to evaluate mast cell activation during the responses to nasal challenge with allergen, cold, dry air, and hyperosmolar solutions. J Allergy Clin Immunol 1992;89:1098-110.

7. Philip G, Jankowski R, Baroody F, Naclerio R, Togias A. Reflex activation of nasal secretion by unilateral inhalation of cold dry air. Am Rev Respir Dis 1993;148:1616-22.

8. Togias A, Lykens K, Kagey-Sobotka A, Eggleston P, Proud D, Lichtenstein L, et al. Studies on the relationships between sensitivity to cold dry air, hyperosmolar solutions and histamine in the adult nose. Am Rev Respir Dis 1990;141:1428-33.

9. Togias A, Proud D, Kagey-Sobotka A, Adams G, Norman P, Lichtenstein L, et al. The osmolality of nasal secretions increases when inflammatory mediators are released in response to inhalation of cold, dry air. Am Rev Respir Dis 1988;137:625-9.
10. Sanico AM, Philip G, Lai G, Togias A. Hyperosmolar saline induces reflex nasal secretions, evincing neural hyperresponsiveness in allergic rhinitis. J Appl Physiol 1999;86:1202-10.

11. Ahern GP, Brooks IM, Miyares RL, Wang XB. Extracellular cations sensitize and gate capsaicin receptor TRPV1 modulating pain signaling. J Neurosci 2005;25:5109-16.

12. Eggleston P, Kagey-Sobotka A, Lichtenstein L. A comparison of the osmotic activation of basophils and human lung mast cells. Am Rev Respir Dis 1987; $135: 1043-8$.

13. Silber G, Proud D, Warner J, Naclerio R, Kagey-Sobotka A, Lichtenstein $\mathrm{L}$, et al. In vivo release of inflammatory mediators by hyperosmolar solutions. Am Rev Respir Dis 1988;137:606-12.

14. Naclerio R, Meier H, Kagey-Sobotka A, Adkinson N, Meyers D, Norman P, et al. Mediator release after nasal airway challenge with allergen. Am Rev Respir Dis 1983;128:597-602.

15. Sanico AM, Stanisz A, Gleeson TD, Bora S, Proud D, Bienenstock J, et al. Nerve growth factor expression and release in allergic inflammatory disease of the upper airways. Am J Respir Crit Care Med 2000;161: 1631-5.

16. Siraganian R. An automated continuous flow system for the extraction and fluorometric analysis of histamine. Anal Biochem 1974;57: 283-94.

17. Imanari T, Kaizu T, Yoshida H, Yates K, Pierce J, Pisano J. Radiochemical assays for human urinary, salivary and plasma kallikreins. In: Pisano JJ, Austen KF, editors. Chemistry and biology of the kallikrein-kinin system in health and disease. DHEW pub \# (NIH) 76-791. Washington (DC): Department of Health,Education and Welfare; 1976. p. 205-13.

18. Proud D, Togias A, Naclerio R, Crush S, Norman P, Lichtenstein L. Kinins are generated in vivo following nasal airway challenge of allergic individuals with allergen. J Clin Invest 1983;72:1678-85.

19. Baumgarten C, Togias A, Naclerio R, Lichtenstein L, Norman P, Proud D. Influx of kininogens into nasal secretions following antigen challenge of allergic individuals. J Clin Invest 1985;76:191-7.

20. Bascom R, Pipkorn U, Lichtenstein L, Naclerio R. The influx of inflammatory cells into nasal washings during the late response to antigen challenge: effect of steroid pretreatment. Am Rev Respir Dis 1988; 138:406-12.

21. Philip G, Sanico A, Togias A. Inflammatory cellular influx follows capsaicin nasal challenge. Am J Respir Crit Care Med 1996;153:1222-9.

22. Linder A. Symptom scores as measures of the severity of rhinitis. Clin Allergy 1988;18:29-37.

23. Weller P. Updates on cells and cytokines: human eosinophils. J Allergy Clin Immunol 1997;100:283-7

24. Baraniuk J, Ali M, Yuta A, Fang S-Y, Naranch K. Hypertonic saline nasal provocation stimulates nociceptive nerves, substance $\mathrm{P}$ release, and glandular mucous exocytosis in normal humans. Am J Respir Crit Care Med 1999;160:655-62.

25. Togias A, Proud D, Kagey-Sobotka A, Norman P, Lichtenstein L, Naclerio R. The effect of a topical tricyclic antihistamine on the response of the nasal mucosa to challenge with cold, dry air and histamine. J Allergy Clin Immunol 1987;79:599-604.

26. Souques F, Crampette L, Mondain M, Vignola A, Chanez P, Bousquet J, et al. Stimulation of dispersed nasal polyps cells by hyperosmolar solutions. J Allergy Clin Immunol 1995;96:980-5.

27. Koskela H, Di Sciascio MB, Anderson SD, Andersson M, Chan HK, Gadalla S, et al. Nasal hyperosmolar challenge with a dry powder of mannitol in patients with allergic rhinitis: evidence for epithelial cell involvement. Clin Exp Allergy 2000;30:1627-36.

28. Hwang SW, Cho H, Kwak J, Lee SY, Kang CJ, Jung J, et al. Direct activation of capsaicin receptors by products of lipoxygenases: endogenous capsaicin-like substances. Proc Natl Acad Sci U S A 2000;97:6155-60.

29. Togias A, Thompson M, Cruz A, Kagey-Sobotka A, Proud D, Lichtenstien L, et al. Less mucosal hydration using a modified protocol for cold, dry air (CDA) nasal challenge enhances histamine (HIST) release. Am Rev Respir Dis 1990;141:758A.

30. Cruz A, Togias A, Lichtenstein L, Kagey-Sobotka A, Proud D, Naclerio R. Local application of atropine attenuates the upper airway reaction to cold, dry air. Am Rev Respir Dis 1992;146:340-6.

31. Assanasen P, Baroody F, Rouadi P, Naureckas E, Solway J, Naclerio R. Ipratropium bromide increases the ability of the nose to warm and humidify air. Am J Respir Crit Care Med 2000;162:1031-7. 
32. Anderson S, Togias A. Dry air and hyperosmolar challenge in asthma and rhinitis. In: Busse WW, Holgate S, editors. Asthma and rhinitis. Oxford: Blackwell Scientific Publications Inc; 2000. p. 1449-68.

33. Freed A, Peters S, Menkes H. Airflow-induced bronchoconstriction: role of epithelium and eicosanoid mediators. J Appl Physiol 1987;62: 574-81.

34. Freed A, Omori C, Schofield B, Mitzner W. Dry air-induced mucosal cell injury and bronchovascular leakage in canine peripheral airways. Am J Respir Cell Mol Biol 1994;11:724-32.

35. Freed A, Wang Y, McCulloch S, Myers T, Suzuki R. Mucosal injury and eicosanoid kinetics during hyperventilation-induced bronchoconstriction. J Appl Physiol 1999;87:1724-33.

36. Ingenito E, Pliss L, Ingram R, Pichurko B. Bronchoalveolar lavage cell and mediator responses to hyperpnea-induced bronchoconstriction in the guinea pig. Am Rev Respir Dis 1990;141:1162-6.

37. Hanes L, Issa E, Proud D, Togias A. Stronger nasal responsiveness to cold air in individuals with rhinitis and asthma, compared with rhinitis alone. Clin Exp Allergy 2006;36:26-31.
38. Assanasen P, Baroody F, Naureckas E, Solway J, Naclerio R. The nasal passage of subjects with asthma has a decreased ability to warm and humidify inspired air. Am J Resp Crit Care Med 2001;164:1640-6.

39. Pliss L, Ingenito E, Ingram R, Pichurko B. Assessment of bronchoalveolar cell and mediator response to isocapnic hypernea in asthma. Am Rev Respir Dis 1990;142:73-8.

40. Larsson L, Hemmingsson P, Boethius G. Self-reported obstructive airway symptoms are common in young cross-country skiers. Scand J Med Sci Sports 1994;4:124-7.

41. Heir T. Longitudinal variations in bronchial responsiveness in crosscountry skiers and control subjects. Scand J Med Sci Sports 1994;4: 134-9.

42. Sue-Chu M, Larsson L, Moen T, Rennard S, Bjermer L. Bronchoscopy and bronchoalveolar lavage findings in cross-country skiers with and without "ski asthma." Eur Respir J 1999;13:626-32.

43. Davis MS, Schofield B, Freed AN. Repeated peripheral airway hyperpnea causes inflammation and remodeling in dogs. Med Sci Sports Exerc 2003;35:608-16. 